\title{
A New Algorithm of Automatic Image Segmentation Based on PCNN
}

\author{
Fan Bin-Wen ${ }^{1, a^{*}}$, Wu Wei ${ }^{1}$ \\ ${ }^{1}$ Harbin Institute of Technology Shenzhen Graduate School, China \\ aww_hit@163.com
}

Keywords: pulse coupled neural network; image segmentation; parameter adaptive setting.

\begin{abstract}
Pulse coupled neural network (PCNN) has a difficult problem to determine the parameters, mainly based on artificial experience. We propose an automatic image segmentation method based on PCNN, which is combining with dynamic threshold and global threshold, reducing manual intervention, realizes the adaptive parameter setting. Finally, the experimental segmentation result of lots of gray images show that the method proposed in this paper can not only achieve automatic image segmentation, but also has strong adaptability.
\end{abstract}

\section{Introduction}

In recent years, pulse coupled neural network (PCNN) is widely used in image processing, and has achieved remarkable results. Image segmentation is a technique to distinguish the different meanings and extract the interested target of image. Particularly, The results of segmentation directly affect the quality of subsequent image analysis. How to effectively and adaptively make the image segmentation has been a difficult problem in image segmentation. According to the image modeling algorithm, the image segmentation methods based on regions [1], edge detection [2] and mathematical morphology have been divided before. For the image segmentation technology based on PCNN, many scholars have put forward various kinds of methods [3,5], such as PCNN with fuzzy $\mathrm{C}$ means [4]. There is also a combination of PCNN and region growing [1], but it requires to manually set the time-variant parameters. In addition, although the pulse coupled neural network (PCNN) model has been widely used in digital image segmentation, it has been a difficult problem to determine the network parameters and the optimum results, mostly by the artificial experience.

Here, we propose a new image segmentation method based on pulse coupled neural network (PCNN). In this method, we improve and simplify the PCNN model, and then combine the dynamic threshold generated by PCNN and the global threshold obtained by OTSU. Then, we determine the best segmentation results by using the maximum entropy criterion.

\section{PCNN Model and Improved}

The pulse coupled neural network is a typical model, which is composed of neurons. Each neuron represents a pixel in the image, thus constituting an image processing system. They satisfy the following equations [1]:

$$
\begin{aligned}
& F_{i j}(n)=e^{-\alpha_{F}} F_{i j}(n-1)+V_{F} \sum_{k, l} M_{i j k l} Y_{k l}(n-1)+S_{i j} \\
& L_{i j}(n)=e^{-\alpha_{L}} L_{i j}(n-1)+V_{L} \sum_{k, l} W_{i j k l} Y_{k l}(n-1) \\
& U_{i j}(n)=F_{i j}(n)\left(1+\beta L_{i j}(n)\right)
\end{aligned}
$$




$$
\begin{aligned}
& E_{i j}(n)=e^{-\alpha_{E}} E_{i j}(n-1)+V_{E} Y_{i j}(n-1) \\
& Y_{i j}(n)=\left\{\begin{array}{c}
1, U_{i j}(n)>E_{i j}(n-1) \\
0, \text { otherwise }
\end{array}\right.
\end{aligned}
$$

The accept domain is divided into two channels, one is linking input channel $\mathrm{L}_{\mathrm{ij}}$, which is used to receive the input information from other neurons, the other is the feedback input channel $F_{i j}$, which is used to receive the external stimulus information $S_{\mathrm{ij}}$. $\alpha_{\mathrm{F}}$ and $\alpha_{\mathrm{L}}$ are the time decay constant, $V_{F}$ and $\mathrm{V}_{\mathrm{L}}$ are amplification coefficient. The weight matrix $\mathrm{M}_{\mathrm{ijkl}}$ and $\mathrm{W}_{\mathrm{ijk}}$ are the linking matrix. The feedback input and the linking input are modulated through linking strength $\beta$ to yield interior active item $U_{i j}$ which is compared with the $E_{i j}$. When the internal active item $U_{i j}$ is larger than the dynamic threshold $\mathrm{E}_{\mathrm{ij}}$, the neuron fire and the output pulse sequence is generated. Eq. (1) (5) demonstrated the image processing of PCNN, which is called the traditional PCNN algorithm.

Because of the too many parameters of traditional PCNN model, it is not good for setting parameters and realizing adaptively segmentation. So this paper uses PCNN's simplify model ${ }^{[1]}$. The feedback input $\mathrm{F}_{\mathrm{ij}}$ only receives the external input $\mathrm{I}_{\mathrm{ij}}$ that the neuron corresponds to the pixel gray value, through the linking matrix $\mathrm{W}$ adjusts the linking input $\mathrm{L}_{\mathrm{ij}}$.

$$
\begin{aligned}
F_{i j}(n) & =I_{i j} \\
L_{i j}(n) & =\sum_{k, l} W_{i j k l} Y_{k l}(n-1)
\end{aligned}
$$

In order to make the threshold traverse any possible gray value and make it suitable for image processing applications, we make threshold use linear decay mode [6]. The attenuation step is D. This paper chooses the dynamic threshold adjustment function is the Eq. (8):

$$
E_{i j}(n)=E_{i j}(n-1)-D+V_{E} Y_{i j}(n-1)
$$

\section{Description of Algorithm}

3.1 Dynamical Threshold and Global Threshold. For an image, if the gray histogram of it has clear two peaks, the global threshold segmentation can obtain a very good segmentation effect. However, when the target image is more complex, and the gray histogram can not see clearly two peaks, the result will not be ideal. We can consider using dynamic threshold to segment the image. But the dynamic threshold segmentation is easy to produce shadow and artificial boundaries. So we should combine the global threshold and dynamic threshold [7].

According to its ability to cause the adjacent neurons with similar inputs to pulse synchronously, PCNN is suitable for image segmentation. The dynamic threshold of PCNN is caused by the stimulation of the adjacent neurons. Because the neuron firing sequence is based on the gray value of the image, so the image can be divided into several regions.

The global threshold is obtained by OTSU. OTSU is adaptive threshold setting method proposed by Japanese scholar in 1979 [8]. The core is in accordance with the gray level of the image, using a threshold divide the image into two parts that is the target and background. The variance of target and background is larger, and the difference between the two parts of the image is larger. The formula for calculating the variance is Eq. (9):

$$
S=W_{A}\left(\mu_{A}-\mu\right)^{2}+W_{B}\left(\mu_{B}-\mu\right)^{2}
$$

The dynamic threshold and global threshold Eq. (10) are used to construct the matrix T, where the $\mathrm{K}$ value ranges from 0 to 1 when difference of the background and the object is large, can select 
the small $\mathrm{K}$ value, and the large $\mathrm{K}$ value can be selected when difference of the background and object are small.

$$
T=k * S+(1-k) * E
$$

Then comparing with gray value of the original image, if the original gray value is greater than the new threshold, the output is 1, conversely, the output is 0, as in Eq. (11).

$$
Y_{i j}(n)=\left\{\begin{array}{c}
1, I_{i j}(n)>T_{i j}(n) \\
0, \text { otherwise }
\end{array}\right.
$$

3.2 Experiment Design. Combined with the above analysis, we can draw the PCNN algorithm for automatic image segmentation process as follows:

(1) Firstly, using the simplified PCNN model to make the first round of iteration, through adaptive search for linear attenuation parameter $\mathrm{D}$, and can set $\mathrm{D}=\mathrm{D}+2$. According to a lot of experiments, the first round of the cycle can be completed within 50 times. According to the maximum entropy evaluation criterion, the iteration number of the best output images is A1.

(2) In the first time, the number of iterations has been determined, use it as the iteration number of the inner cycle and set the $\mathrm{D}=\mathrm{i}$ that $\mathrm{i}$ is the iterations number of the external cycle. The external cycle iteration number A2 can be set when the output has maximum entropy.

(3) Repeat step 2, but the number of external cycle iterations is set to A2, and then the optimal segmentation results are obtained.

\section{The Experiment of Analysis}

According to the above model and algorithm, a lot of experiments are carried out to prove the reliability of the algorithm. The Girl and Rice images are selected as the target images and compared with the OTSU and [9]. The experimental environment is Matlab 7. 0. In the experiment, the parameters of PCNN model: $\mathrm{D}=0, \beta=0.02, \mathrm{~K}=0.7$, the weight matrix, as in Eq. (12).

$$
W_{i j k l}=\left[\begin{array}{ccc}
0.5 & 1 & 0.5 \\
1 & 0 & 1 \\
0.5 & 1 & 0.5
\end{array}\right]
$$
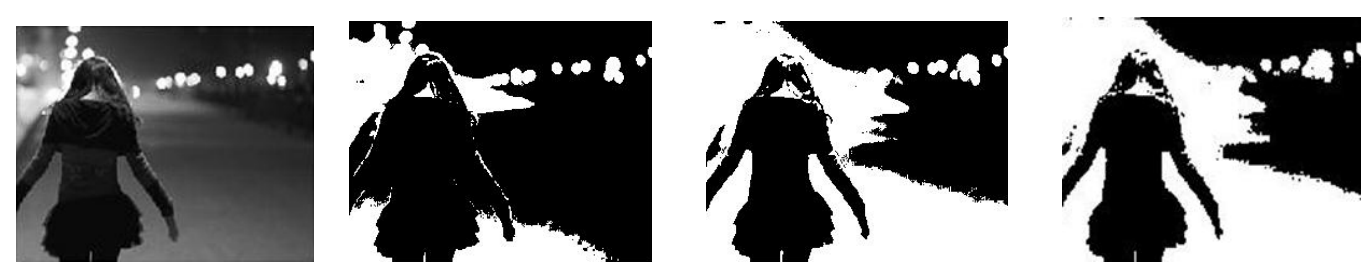

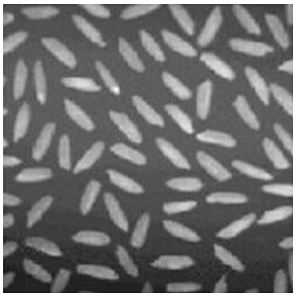

(a)

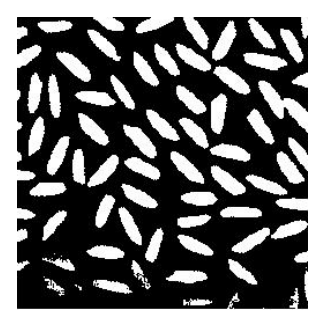

(b)

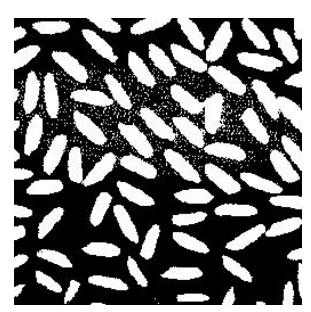

(c)

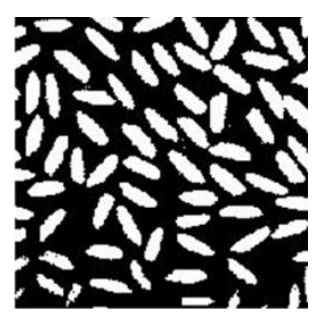

(d)

Fig 1. Segmentation results of Girl, Rice and Man: column (a)original input image. column (b)result of Otsu. column (c) result of BPCNN. column (d)result of this paper.

(1) In Girl image, the girl next to the road, Otsu produces the phenomenon of over-segmentation, BPCNN results is similar to the results of this paper, but tBPCNN still has some shortcomings, 
between road and arm has over-segmentation phenomenon. The algorithm of this paper has a good result for segmentntation of girl body, and is good to remove background and get a better segmentation result.

(2) In Rice image, the segmentation effect of Otsu is not good, which is because the bottom of the source image of the pixel value of rice is lower than the most of the target gray level, then make them into the background by false. The algorithm of this paper is very good to overcome this problem. Use the BPCNN method will produce more noise in the upper part of the image.

From the experimental results, it can be seen that the method this paper proposed is better than the method proposed by [9]. Compared with the traditional Otsu method, the this paper method has better universality.

\section{Conclusions}

PCNN has wide application in image processing and signal processing. In this paper, a self-adaptive image segmentation method is proposed which combines the global threshold and the dynamic threshold based on the simplified PCNN. This method make use of the global threshold and dynamic threshold, and has a certain universality for gray image. PCNN automatic image segmentation is implemented, and the application domain of PCNN is extended.

\section{References}

[1] Stewart, R. D., I. Fermin, and M. Opper. Region growing with pulse-coupled neural networks: an alternative to seeded region growing. Neural Networks IEEE Transactions on 13.6(2002) $1557-1562$.

[2] Deng, Xiangyu, and M. A. Yide. PCNN Model Analysis and Its Automatic Parameters Determination in Image Segmentation and Edge Detection. Chinese Journal of Electronics 01.1(2014) 97-103.

[3] Tao, Zhongyu, et al. Image segmentation based on PCNN model. Wavelet Active Media Technology and Information Processing (ICCWAMTIP), 2014 11th International Computer Conference on IEEE, (2014)230 - 233.

[4] SUBASHINI M M, SAHOO S K. Brain tumour detection using Pulse coupled neural network (PCNN) and back propagation network; proceedings of the Sustainable Energy and Intelligent Systems (SEISCON 2012), IET Chennai 3rd International on, F, 2012 [C].

[5] CHEN Y, PARK S K, MA Y, et al. A new automatic parameter setting method of a simplified PCNN for image segmentation. J. IEEE Transactions on Neural Networks,22(6) (2011) 880 - 92.

[6] Chen, Yuli, et al. A new automatic parameter setting method of a simplified PCNN for image segmentation. IEEE Transactions on Neural Networks 22.6 (2011) 880 - 892.

[7] Qi-Yuan, T. U., W. U. Jian-Hua, and G. J. Wan. Image Segmentation Using Dynamic Threshold Combinedwith Global Threshold. Journal of Nanchang University (2002).

[8] OTSU N. A Threshold Selection Method from Gray-Level Histograms . J. Systems Man \& Cybernetics IEEE Transactions on, 9(1) (1979) 62-6.

[9] Yang, Bin, and W. U. Hao-Jiang. Image Segmentation Based on Bidirectional Search Pulse Coupled Neural Network. Techniques of Automation \& Applications (2009). 